

\title{
Rahmenbedingungen und Betreuung: ein ganzheitlicher Ansatz
}

\author{
A. Pelet, B. Broers, M. Monnat
}

\section{Im Spital}

Die Spitalbetreuung eines drogenabhängigen Patienten ist zuweilen für Ärzte wie Pflegepersonal schwierig. Auf Seiten des medizinischen Teams bestehen viele Vorbehalte (die Befürchtung manipuliert zu werden, ungenügende Kenntnisse über eine strukturierte Betreuung von drogenabhängigen Patienten, Vermeidungshaltung, Vorurteile, Misstrauen, moralische Verurteilungen) [1]. Der Patient seinerseits hat möglicherweise grosse Angst, denn im Vergleich zu einem nicht drogenabhängigen Patientenkollektiv desselben Alters haben Suchtkranke bereits viele Freunde im Spital sterben sehen. Er hat Angst, auf Entzug zu kommen und zu leiden (Schmerzen werden bei Drogenabhängigen häufig nur unzureichend behandelt [2]), und hat eventuell bereits erlebt, beim Behandlungspersonal auf Ablehnung und Verachtung zu stossen [1]. Die in diesem Kapitel behandelten praktischen Aspekte stellen sogenannte «niederschwellige» Ansätze dar, die sich im Wesentlichen an schwer zu erreichende Patienten richten.

\section{Aufbau einer Beziehung}

Wie bei allen Patienten ist es wichtig, eine Vertrauensbeziehung herzustellen. Bei drogenabhängigen Menschen ist der Angstfaktor nicht zu unterschätzen, der sich zuweilen in aggressivem oder misstrauischem Verhalten äussern kann. Es ist wichtig, dem Patienten zu sagen, dass er alle Schwierigkeiten sowohl auf persönlicher Ebene als auch im Umgang mit dem Behandlungspersonal ansprechen darf, und ihm zu versichern, dass man ihn als Patienten respektieren wird. Bei dieser Gelegenheit kann man ihn auch darauf aufmerksam machen, dass dieser Respekt reziprok sein muss, also auch das Behandlungsteam respektiert werden muss. Zuweilen ist es sinnvoll, ihn an die häufig 
stillschweigenden Verhaltensregeln zu erinnern (Einhaltung der Nachtruhe, Rauchen nur an den dafür vorgesehenen Orten, Respekt gegenüber dem Personal, Anwesenheit zu den Essenszeiten, während der Visite und für die pflegerischen Massnahmen etc.). Konflikte, bei denen es zu übertriebenen (zuweilen unangemessenen) Forderungen von Seiten des Behandlungspersonals und aggressivem Verhalten auf Patientenseite kommt, aber auch das gegenteilige Extrem einer zu permissiven Haltung aus Überdruss oder Furcht vor Konflikten, sollten vermieden werden. In diesem Kontext ist nicht zu vergessen, dass bei drogenabhängigen Patienten häufig eine psychiatrische Komorbidität vorliegt [3]. Idealerweise fürchtet sich die behandelnde Person nicht, den Patienten zu konfrontieren, und bemüht sich aber gleichzeitig, die therapeutische Beziehung aufrechtzuerhalten.

\section{Einfühlsame und unvoreingenommene Betreuung}

Drogenabhängige Patienten rufen in einem Pflegeteam häufig starke emotionale Reaktionen hervor (Ablehnung, Mitleid, Uneinigkeit im Behandlungsteam in Bezug auf ein Verhalten etc.). In solchen Situationen können Aussprachen (in schwierigen Fällen mit Hilfe eines Supervisors) hilfreich sein. Ein stabiles Team mit geringer Personalfluktuation wirkt sich förderlich aus. Kleine Gesten (den zuständigen Sozialarbeiter des fürsorgeabhängigen Patienten um etwas Taschengeld für Zigaretten oder die Fernsehermiete bitten oder den Seelsorger für ein Gespräch anfordern etc.) verbessern oft deutlich die Atmosphäre und erleichtern den Krankenhausaufenthalt.

\section{Schmerz}

Schmerzen müssen unverzüglich bei der Aufnahme evaluiert und behandelt werden (siehe Kapitel «Schmerz und Methadon»).

\section{Beginn einer Substitutionsbehandlung}

Bei hospitalisierten Patienten mit einer Opiatabhängigkeit, die nicht (mit Methadon oder anderen Mitteln) substituiert ist, ist es wichtig, unbedingt eine Substitutionsbehandlung zu beginnen. Dabei ist nicht zu vergessen, vor Aufnahme der Substitution einen Urintest durchzuführen! (CAVE: ausländische Patienten ohne Niederlassungspapiere: diese Fälle sind mit einem spezialisierten Zentrum abzusprechen). Wird die Behandlung erstmalig aufgenommen, muss unverzüglich ein Bewilligungsantrag an den Kantonsarzt gestellt und mit dem Patienten ein Vertrag geschlossen werden. Dabei variieren die Regelungen gemäss den lokalen Empfehlungen. Die Entlassung des Patienten bedarf einer guten Vorbereitung und der weiterbetreuende Arzt oder die entsprechende Einrichtung sind stets vor der Entlassung zu unterrichten. (Informationen über die Dosierung der Substitutionssubstanzen sind bspw. auf der Website http://www.romandieaddiction.ch zu finden.) 


\section{Anpassung der Substitutionsbehandlung (Methadon und andere Substanzen)}

Bei Anzeichen eines Entzugssyndroms oder einer Intoxikation ist eine Anpassung der Dosierung erforderlich. Ferner kann eine Erhöhung der Dosis notwendig sein, wenn der Patient nicht stabilisiert werden kann und zusätzlich zum verordneten Methadon weiterhin Heroin konsumiert. Allerdings muss jede Änderung der Dosierung in Absprache mit dem Patienten und dem externen Betreuungsumfeld erfolgen (CAVE: Medikamenteninteraktion mit Methadon, wenn eine Substanz mit dem Cytochrom P450 und insbesondere CYP2D6 und CYP3A4 interagiert) [4].

\section{Methadon}

Beruhigen Sie den Patienten in Bezug auf sein Methadon und versichern Sie ihm, dass er es zur gewohnten Dosis und nach festem Zeitplan erhält. Möglicherweise hat der Patient bereits «Machtspiele» erlebt, bei denen ihm seine Medikation vom medizinischen Personal verweigert wurde. Eine Methadonbehandlung darf vom Behandlungspersonal nicht instrumentalisiert werden, um den Patienten "zu belohnen» oder "zu bestrafen». Es ist so rasch wie möglich der Kontakt zum behandelnden Arzt oder der zuständigen Apotheke herzustellen, um die vom Patienten angegebene Dosierung und die Begleitmedikation zu überprüfen (namentlich Benzodiazepine). Erscheint dem Arzt im Spital die vom Patienten angegebene Dosierung nicht korrekt, kann er das Methadon in den ersten Tagen fraktionieren, um eine eventuelle Überdosierung zu verhindern. Mit Ausnahme von Notfällen ist der einzige Grund für eine Fraktionierung der täglichen Methadondosis der Nachweis eines schnellen Metabolismus (ein pharmakokinetischer Test und die Bestimmung des Methadonblutspiegels geben Aufschluss [4]). Bei Verdacht auf eine schnelle Metabolisierung (Kontakt mit dem behandelnden Arzt aufnehmen) kann dieser Test problemlos im Rahmen der Hospitalisation durchgeführt werden.

Es wird davon abgeraten, während der Hospitalisation ohne triftigen Grund Psychopharmaka zu verabreichen (insbesondere Benzodiazepine) oder sie bei der Entlassung zu verordnen.

\section{Andere Abhängigkeiten}

Als erster Schritt muss der Arzt eine häufig vorliegende Mehrfachabhängigkeit abklären. Bei einer Kokainabhängigkeit bedarf es keiner Substitution. Allerdings kann es zu Phasen mit sogenanntem «Craving» kommen (unbezwingbares Verlangen, Kokain zu konsumieren), die möglichst mit nicht medikamentösen Methoden angegangen werden sollten (zum Beispiel mit Sport, Yoga oder Entspannung). Ausserdem ist zu untersuchen, ob eine Alkoholabhängigkeit vorliegt, die ggf. über einige Tage mit Benzodiazepinen mit kürzerer Wirkungsdauer (vom Typ Oxazepam; Seresta ${ }^{\circledR}$ ) und in abnehmender Dosierung zu behandeln ist. 
Ferner ist eine mögliche Benzodiazepin-Abhängigkeit zu erfassen und die Notwendigkeit einer Substitution zu erwägen, da bei einem Benzodiazepin-Entzug epileptische Anfälle auftreten können und die Gefahr besteht, dass der Patient bei Entzugserscheinungen das Krankenhaus überstürzt verlässt. Die Mittel der Wahl sind Benzodiazepine mit längerer Halbwertzeit, wie beispielsweise Clonazepam (Rivotril ${ }^{\oplus}$ ) oder Clorazepam (Tranxilium ${ }^{\circledast}$ ). Substanzen mit kurzer Halbwertzeit wie Midazolam (Dormicum ${ }^{\circledR}$ ) sind zu vermeiden, da sie ein erhöhtes Abhängigkeitsrisiko bergen und eine Verlagerung des Konsums droht. Es muss ein Therapieplan mit langsamer Dosisreduktion und sinnvollen Zielsetzungen erarbeitet und mit dem Patienten besprochen werden. Wünschenswert ist es, eine Benzodiazepin-Substitution unter Beteiligung des nachbehandelnden Arztes zu beginnen, der über jede Änderung der Behandlung rasch informiert werden muss.

\section{Konsum illegaler Substanzen}

Der Konsum illegaler Substanzen ist verboten. Dies ist dem Patienten nochmals deutlich zu machen. Einige Spitäler sind verpflichtet, jeden illegalen Konsum den Behörden zu melden. Allerdings muss sich das Spital mit dem externen Betreuungsumfeld abstimmen und darf sich nicht auf repressive Massnahmen beschränken. Es ist wichtig, den Patienten insbesondere für das Problem der gebrauchten Spritzen zu sensibilisieren. Besser ist es, wenn der Patient seine Spritzen in dafür vorgesehenen Behältern entsorgt, als dass er sie unter dem Bett versteckt! Die Betreuung im Spital sollte mit der externen Betreuung kohärent sein (schweizerische Vier-Säulen-Politik: Prävention, Repression, Therapie und Überlebenshilfe).

\section{Abschluss eines Vertrags mit dem Patienten}

Die Erstellung eines Vertrags kann zuweilen helfen, eine schwierige Betreuung zu strukturieren und für beide Seiten - Patient wie Behandlungspersonal - die an den Patienten gestellten Anforderungen eindeutig zu definieren. Dabei ist das Anforderungsniveau aber auf die Möglichkeiten des Patienten abzustimmen und eine mögliche psychiatrische Komorbidität sollte Berücksichtigung finden. (Beispiel: Es ist illusorisch, von einem verängstigten Heroin- und Kokainabhängigen zu verlangen, das Zimmer nicht zu verlassen, sei es auch nur, um eine Zigarette zu rauchen!)

In einem solchen Betreuungsvertrag sind auch mögliche Regelüberschreitungen vorwegzunehmen sowie die entsprechenden realistischen Sanktionen gegen den Patienten festzuhalten. Diese müssen stets gegen die Wichtigkeit, den Patienten im Spital halten und behandeln zu können, abgewogen werden. (Beispiel: Ist es sinnvoll, einen Patienten mit einer Endokarditis zu entlassen, wenn die gestellten Anforderungen für ihn nicht erfüllbar sind?) Bei Schwierigkeiten sollte man nicht zögern, Rat und Hilfe eines Spezialdienstes in Anspruch zu nehmen. 


\section{Betreuung im Netzwerk}

Die Betreuung eines drogenabhängigen Patienten erfolgt im Wesentlichen in einem Netzwerk. Daher muss während der stationären Behandlung unbedingt der Kontakt zum ambulanten Betreuungsnetz (behandelnder Arzt, spezialisiertes Zentrum, Sozialarbeiter) aufrecht erhalten werden. Die an der ambulanten Betreuung Beteiligten können dem Behandlungspersonal im Spital eine grosse Hilfe sein. Die Entlassung des Patienten muss unbedingt im Voraus organisiert werden, damit dieser ohne Unterbrechung ambulant weiter betreut werden kann.

\section{Ambulante Betreuung}

Die ambulante Betreuung von drogenabhängigen Patienten ist eine langfristige Arbeit - wie bei allen chronisch kranken Menschen. Dabei können zahlreiche Probleme auftreten: Erschöpfung der Ärzte, hoher Zeitaufwand, Probleme beim Aufbau eines Netzwerks, keine ausreichenden Kenntnisse in der Behandlung und den Grundregeln einer Betreuung Drogenabhängiger [5-7]. Eine adäquate Ausbildung gibt dem Arzt mehr Sicherheit im Umgang mit den drogenabhängigen Patienten. Überdies ermöglicht die Arbeit im Netzwerk in schwierigen Situationen gegenseitige Hilfe und verhindert ein Auslaugen des Behandelnden.

Sich fortbilden im Suchtbereich. Die Betreuung eines Suchtpatienten erfordert Kenntnisse in den Substitutionsprodukten, ihrer Dosierung, den Wechselwirkungen und der Auswahl des Produkts je nach Kontext. Informationen sind im Internet zuweilen in interaktiver Form erhältlich, bspw. unter (http://www.romandieaddiction.ch oder http://www.ssam.ch).

Teilnahme an einer Gruppe von Ärzten, die Drogenabhängige betreuen, kann wichtige Fortbildungs- und Unterstützungsmöglichkeiten bieten (Informationen über COROMA unter http://www.romandieaddiction. ch).

Einbezug (oder Schaffung) des Netzwerkes, das den Patienten umgibt. Die soziale Situation eines drogenabhängigen Patienten hat in den meisten Fällen Einfluss auf dessen Behandlung. Dabei muss der Arzt besonders die Lebensumstände des Patienten berücksichtigen (Beispiel: schwierige Wohnsituation, fehlendes Einkommen, Prostitution etc.). Die Betreuung ist in höchstem Masse multidisziplinär [8]! Kontakte zu den Sozialarbeitern sind wichtig und ermöglichen häufig die Strukturierung und Koordinierung der Betreuung. 
In allen Kantonen wurden von den Kantonsärzten Empfehlungen für Ärzte ausgearbeitet, die eine Substitutionsbehandlung mit Methadon verschreiben. Sie enthalten die praktischen Modalitäten für die Methadonabgabe und die jeweiligen Pflichten der beteiligten Patienten und Ärzte. Es ist wichtig, diese Empfehlungen beim zuständigen Kantonsarzt anzufordern.

Bei Problemen sollte sich der Arzt an ein spezialisiertes Zentrum wenden (Beratung, Hilfe) oder an Kollegen aus der Suchtmedizin.

\section{Referenzen}

1 Merrill JO, Rhodes LA, Deyo RA, Marlatt AA, Bradley KA: Mutual mistrust in the medical care of drug users: The keys to the 'narc' cabinet. J Gen Intern Med 2002;17:327-333.

2 Jamison RN, Kauffman J, Katz N: Characteristics of methadone maintenance patients with chronic pain. J Pain Symptom Manage 2000;19:53-62.

3 Von Limbeek J, Wouters L, Kaplan CD, Geerlings PJ, Von Alem V: Prevalence of psychopathology in drug-addicted Dutch. J Subst Abuse Treat 1992;9:43-52.

4 Eap CB: Nouvelles études psychopharmacologiques sur la méthadone: Implications pour le traitement de la dépendance aux opiacés. Rev Méd Suisse Rom 2000;120:111116.

5 Detzer E, Muller B, Carlin A: Identifying and treating the drug-misusing patient. Am Fam Physician 1977;16:181-186.

6 McGillion J, Wanigaratne S, Feinmann C, Godden T, Byrne A: GPs' attitudes towards the treatment of drug misusers. Br J Gen Pract 2000;50:385-386.

7 Saitz R, Friedmann PD, Sullivan LM, Winter MR, Lloyd-Travaglini C, Moskowitz MA, Samet JH: Professional satisfaction experienced when caring for substance-abusing patients. J Gen Intern Med 2002;17:373-376.

8 Eidgenössische Betäubungsmittelkommission: Methadonbericht. Bern, Bundesamt für Gesundheit, 1995. 
